\title{
Indicadores y control estadístico para el seguimiento y evaluación de preferencias de aprendizaje de estudiantes universitarios
}

\section{Indicators and statistical control in monitoring and assessing university students' learning preferences}

\author{
Olga María Alegre de la Rosa \\ Universidad de La Laguna, La Laguna, España \\ oalegre@ull.edu.es \\ Luis Miguel Villar Angulo \\ Universidad de Sevilla, Sevilla, España \\ mvillar@us.es
}

\begin{abstract}
Resumen
Introducción. Las universidades españolas adoptan indicadores de resultados como práctica destacada de renovación institucional. En este estudio se analizan indicadores de resultados universitarios (tasas de éxito, eficiencia, graduación, abandono y rendimiento de los estudiantes universitarios) de distintos campos del conocimiento, para contrastarlos con la opinión de las comisiones de calidad de los distintos centros universitarios. Método. Se utilizaron métodos mezclados: un análisis cuantitativo de las medias de los porcentajes obtenidos para los diferentes campos del conocimiento para los cinco indicadores de resultados y un análisis cualitativo mediante un DAFO (debilidades, amenazas, fortalezas y oportunidades) con la opinión de las comisiones de calidad de las titulaciones en seis dimensiones de contenido: programa formativo, infraestructura y recursos, estudiantes, profesores, y elementos institucionales. Resultados. Ciencias de la Salud se muestra como la única rama del conocimiento que mejora en tasa de éxito en tres años, sin embargo Ciencias Sociales y Jurídicas e Ingeniería y Arquitectura mejoran en eficiencia. La rama de Artes y Humanidades tiene el porcentaje de deserción más alto. Discusión. Encontramos similitudes con otros estudios en la dirección de la mejora en tasas de éxito en Ciencias de la Salud, que contrasta con las tasas de deserción de Ingeniería y Arquitectura, así como la baja tasa de finalización de estudios en el grado de Historia del Arte. Conclusiones. Se plantea una revisión longitudinal de la metodología docente de las asignaturas, la formación en docencia universitaria, el establecimiento de un plan de mejora para el aprendizaje mezclado y a distancia, y el rendimiento de los estudiantes.
\end{abstract}

Palabras clave

Indicadores, éxito, eficiencia, graduación, abandono, rendimiento, análisis DAFO, aprendizaje mezclado y a distancia.

\footnotetext{
Abstract

Introduction. Spanish universities adopt performance indicators as a prominent practice of institutional renewal. In this study, university performance indicators (students' success rates, efficiency, graduation, dropout and attainment) of different fields of knowledge are analyzed to compare them with the opinion of the quality commissions of the different university centers. Methodology. Mixed methods were used: a quantitative analysis of percentage means of the different fields of knowledge for the five university performance indicators and a qualitative analysis by means of a SWOT (weaknesses, threats, strengths and opportunities) with the opinion of the quality commissions in six dimensions of content: training program, infrastructure and resources, students, teachers, and institutional elements. Results. Health Sciences are shown as the only branch of knowledge that improves the success rate in three years, however Social Sciences and Law and Engineering and Architecture improve efficiency. The Arts and Humanities branch has the highest dropout rate. Discussion. We find similarities with other studies in the success rates improvement in Health Sciences, which contrasts with the dropout rates of Engineering and Architecture, as well as the low rate of graduation in the History of Art degree. Conclusions. It is proposed a longitudinal review of the instructional methods, training in university teaching and a plan for improvement distance learning progress and enhancing performance of trainees. Key words Indicators, student success, efficiency, completion, dropout, attainment, SWOT framework, blended and distance learning.
} 


\section{Introducción}

Las universidades españolas adoptan índices alternativos de responsabilidad institucional como una innovación compuesta de intervenciones mensurables para la profesión docente, una alteración ordenada de preferencias de aprendizaje para los estudiantes y una revelación coordinada de iniciativas para la renovación institucional. La educación universitaria está implicada en debates de política educativa para la identificación y medición de estándares que mejoren el ambiente y la vida en la educación superior. La búsqueda de una reorganización institucional mejorada surge dentro de los recintos universitarios europeos como parte del reiterado impulso para proporcionar una enseñanza de calidad que produzca convincentes resultados de aprendizaje a corto y largo plazo. Una mirada al aprendizaje universitario descubre la profunda exposición a una enjundia de ideas evaluativas, que involucra a muchas personas, matices de experiencias y trayectorias mixtificadas de evaluación dentro y fuera del aula. Al tiempo, los litigios por el rendimiento económico en la sociedad acentúan la desconexión entre la institución académica y la sociedad. En efecto, parece que la educación superior está pugnando por conectar con el paradigma de la eficiencia empresarial, mientras que los profesores, frente a los equipos de gobierno universitario, se encuentran reticentes a usarlo. Debido a presiones de los gobiernos nacional y autonómico sobre los objetivos orientados al rendimiento de estudiantes, a la medición de la calidad universitaria basada en indicadores y a otros métodos basados en fórmulas para la financiación universitaria, la Universidad de La Laguna (ULL) investiga la armonización de la política europea de aseguramiento de la calidad de la educación pública y los procesos académicos internos de gestión estratégica. Además, la Agencia Nacional de Evaluación de la Calidad y Acreditación (ANECA) influye en todas las universidades españolas en general y en los órganos de gobierno de la ULL, en particular, para acreditar todos los grados académicos, involucrando a tal efecto a comisiones internas de calidad, y a otros beneficiarios externos.

\subsection{Revelación de la tasa de éxito de estudiantes}

El éxito del estudiante tiene implicaciones para la política curricular universitaria. Es importante porque impide el abandono de los estudiantes en asignaturas y grados, y evalúa la eficacia académica del profesorado (Howell y Buck, 2012). Cuando existe una congruencia entre los factores universitarios de espacio, tiempo, instalaciones e inversión económica, y las necesidades, intereses y preferencias de los estudiantes, se alcanza más fácilmente la satisfacción estudiantil y se obtiene con más claridad el éxito profesional (Bowman, 2014). Además, la aceptación intrínseca de un estudiante supone un cierto sentido positivo de cumplimiento. Ciertamente la satisfacción intrínseca es un dato importante en la forma en que los alumnos reconocen su éxito en la carrera universitaria (Dumford y Miller, 2015).

\subsection{Verificación de la tasa de eficiencia de estudiantes}

El movimiento social de responsabilidad universitaria exige paralelamente respuestas adecuadas al concepto de autoeficacia universitaria. Esta cuestión teórica resulta intrincada por los diferentes tipos de escalas que los defensores y detractores por igual han utilizado como marco conceptual para dirimir su significado. Como tal, la autoeficacia es un concepto organizador que abarca el apoyo académico y social, y las iniciativas de crecimiento personal. Facilitado este consenso, Çelik (2015:107) está de acuerdo con la siguiente definición de autoeficacia: "Confianza percibida de uno mismo para realizar con éxito una tarea académica particular". Una idea modulada del papel prospectivo de la autoeficacia universitaria es que hay un conjunto de ideas que los estudiantes de primer año deben tener en común, como la persistencia en el aprendizaje y el éxito académico (Wright, Jenkins-Guarnieri y Murdock, 2012). Algunos líderes universitarios están de acuerdo en

Indicadores y control estadístico para el seguimiento y evaluación de preferencias de aprendizaje de estudiantes universitarios. Olga María Alegre de la Rosa y Luis Miguel Villar Angulo. 
que los estudiantes universitarios tienen que completar de manera eficiente sus grados, adquirir competencias profesionales para participar en la mejora económica del país y desarrollar un futuro mejor para las familias y la comunidad (Morris, 2012). Sin embargo, otros investigadores asocian el éxito académico con altos niveles de ansiedad, estrés y enfermedad de los estudiantes. Por otra parte, el aplazamiento de los estudios universitarios refleja un formidable desafío para la renovación universitaria. En medio de estas disquisiciones, sigue teniendo actualidad la afirmación de Hen y Goroshit (2014: 116): "La dilación académica es un impedimento para el éxito académico de los estudiantes". En otro ámbito de estudio, la autoeficacia comprende una variedad de puntos de vista psicológicos en los programas de enseñanza en línea. De acuerdo con Vayre y Vonthron (2016), las cuestiones de autoeficacia de los estudiantes importan porque abordan marcos perceptuales genuinos sobre la implicación de los alumnos y persiguen una preparación eficaz para el aprendizaje en línea. También importa subrayar el apoyo social del profesorado para alcanzar metas ineludibles de compromiso académico.

\subsection{Dilucidación de la tasa de graduación de estudiantes}

¿Cuál es la mejor manera que tienen los estudiantes de graduarse en la educación superior? Los estudiantes universitarios tienen más probabilidades de completar puntualmente un grado académico si lo seleccionan adecuadamente y desarrollan desde el principio una estrategia académica, tienen un itinerario bien definido de los créditos que necesitan para completar el grado, y reciben un estímulo tutorial y una supervisión para ayudarlos a mantenerse en la titulación. De hecho, los políticos y los investigadores han identificado y reconocido las preocupaciones por las tasas de graduación de los estudiantes, ya que "un 30\% de los individuos se arrepienten de su titulación 5 años después de la graduación" (Kucel y Vilalta-Buf, 2013: 576). Una contribución esencial de la educación universitaria es la integración de experiencias curriculares que conjugan elementos imaginativos y apuntes realistas. Este resultado de compromiso formativo se puede producir cuando los alumnos participan en diversas experiencias fuera de clase, incluidas una ocupación dentro o fuera del campus, cooperaciones en residencias, así como aportaciones en asociaciones y sociedades. En consecuencia, y alineado con el relato anterior, Hu (2011: 105) halló: "La relación entre la participación y la permanencia de los estudiantes en la universidad revela que tiene fuertes implicaciones para la investigación de los estudiantes universitarios y la política institucional y la práctica". Paralelamente, Elliott (2016: 427) concluyó en su trabajo: "Los resultados también demostraron que los estudiantes de universidades altamente selectivas con mayores percepciones de autoeficacia académica tenían más probabilidades de ser retenidos". Otro aspecto específico es que un aumento en el tiempo de graduación se ha asociado con una acumulación más lenta de los créditos de un grado. En una investigación española, los estudiantes de clase trabajadora tendían a seleccionar títulos universitarios que eran más fáciles de completar y con oportunidades profesionales más claras y apropiadas para los graduados (Troiano y Elias, 2014). Parece colegirse de lo anterior que los estudiantes que se gradúan más lentamente tienen más dificultades a la hora de adaptarse a un mercado laboral orientado a trabajadores con formación universitaria. En un contexto de minoría extranjera, el estudio de Contreras y Contreras (2015) insinuó que los estudiantes latinos norteamericanos y sus compañeros mostraron menores tasas de graduación universitaria, debido parcialmente a medidas inapropiadas de los datos sobre el éxito.

\subsection{Especificación de la tasa de abandono de estudiantes}

Los estudiantes universitarios que han terminado carreras de cuatro años invierten tiempo, esfuerzo y recursos financieros en su formación personal. Pero muchos de ellos no tienen éxito y se retiran de la universidad sin terminar los créditos en los que se matriculan. Algunos estudios españoles de investigación comparten el fenómeno del abandono universitario. En el caso de la Universidad de

Indicadores y control estadístico para el seguimiento y evaluación de preferencias de aprendizaje de estudiantes universitarios. Olga María Alegre de la Rosa y Luis Miguel Villar Angulo. 
Málaga, Lassibille y Navarro-Gómez (2008: 103) realizando un análisis de sondeo concluyeron que "los estudiantes que se retrasan en la entrada a la educación superior, así como los estudiantes que no consiguieron el curso que querían, están en mayor riesgo de dejar el programa". En consonancia con ese resultado, Arce, Crespo y Míguez-Álvarez (2015: 262) declararon: "Uno de cada cinco estudiantes abandonan la universidad". Por lo tanto, la tasa de abandono es un indicador significativo para estudiar signos y factores de riesgo que tienen en cuenta a estudiantes que comienzan, pero que no terminan sus carreras académicas. De acuerdo con algunas investigaciones alemanas, "las estrategias para apoyar el éxito académico se concentran sólo en ciertos tipos de abandonos. No toman en cuenta el hallazgo empírico de que no hay un solo tipo dominante de deserción, sino varios que son de una importancia similar" (Heublein, 2014: 511). En consecuencia, ¿qué tipos de estudiantes universitarios abandonan los títulos universitarios o se desconectan de los grados? Hay elementos que se correlacionan con la terminación de un grado. Donhardt (2013: 207) ha estudiado este problema y ha encontrado ciertos factores, tales como "hacer una parada en los estudios, asistir a clases de perfeccionamiento, recibir un suspenso, abandonar estudios a tiempo parcial, y dejar plantadas las clases", mientras que Arce, Crespo y Míguez-Álvarez (2015: 262) resumieron los motivos de abandono de los estudios universitarios hallados en otras investigaciones: "incompatibilidad con el trabajo, economía, universidad (medio ambiente, profesores y dificultades) y así sucesivamente".

\subsection{Explicación de la tasa de rendimiento de estudiantes}

Un análisis cuantitativo introduce diversas variables (grados, persistencia, satisfacción, etc.) en la explicación de las causas que podrían conducir a drásticas divergencias en los logros y realizaciones de los estudiantes. Mientras que los resultados de investigación de Mihaljevic (2008) indicaron que los estímulos de los compañeros y los niveles de las tareas de los estudiantes tenían un peso positivo y estadísticamente significativo en el rendimiento de los alumnos, Raynor (2016) ha retado la cuestión generalmente aceptada de que las calificaciones valiosas y el esfuerzo duro son las características más importantes para obtener un rendimiento educativo superior. La revisión de la literatura aporta sistemáticamente ejemplos descriptivos, correlacionales y causales de desigual amplitud y profundidad para la revelación de la trama del rendimiento. Distintos investigadores asocian las habilidades de pensamiento de orden superior y el autocontrol como piezas psicológicas contribuyentes al rendimiento del estudiante (Ghanizadeh, 2016). Otros estudios han establecido una fuerte conexión entre la peculiar asistencia a clase y el rendimiento (Burd y Hodgson, 2016). Según Pike, Hansen y Childress (2014), numerosas medidas de las características de los estudiantes, prácticas de bachillerato, creencias universitarias, y particularidades iniciales de matriculación se relacionaron constantemente con el rendimiento en una titulación. Así, una de las características demográficas investigadas del estudiante es el grupo étnico. En las instituciones de educación superior del Reino Unido, la participación estudiantil en actividades educativas es una piedra angular del rendimiento de los alumnos (Stevenson, 2012; Richardson, 2012). Aceptado el hallazgo, cualquier gobierno universitario debería medir el nivel de implicación de los estudiantes en eventos y sus propuestas de actuación escénica en ámbitos multidisciplinares del campus (Nelson, Quinn, Marrington y Clarke, 2012). Estas piezas de resultados de investigación sobre variables académicas de los estudiantes diseccionan factores psicológicos y sociales que conducen a un rendimiento universitario desigual. En fin, a pesar de que una alta proporción de estudiantes de educación superior está terminando de manera eficiente sus cursos y grados, se puede aumentar el mantenimiento de los alumnos aún más con políticas y prácticas complementarias que sumerjan a los estudiantes universitarios en un lenguaje social comprometido y en una cultura académica interactiva y experiencial (El Hassan, 2014).

Indicadores y control estadístico para el seguimiento y evaluación de preferencias de aprendizaje de estudiantes universitarios. Olga María Alegre de la Rosa y Luis Miguel Villar Angulo. 


\subsection{Aprendizaje mezclado y a distancia}

La separación de un profesor y un estudiante durante la mayoría del tiempo invertido en un proceso instructivo, la inversión en una tecnología por medio de la cual se posibilita el contenido de las materias a los estudiantes, la provisión de un sistema de comunicación entre los agentes del proceso instructivo y la influencia de una organización que sustenta la estrategia evaluativa son características que denotan un aprendizaje a distancia (Al-Arimi, 2014). Conocidas estas premisas, la educación a distancia a nivel superior debe solventar algunas debilidades que algunos autores han percibido en trabajos de investigación aplicada. Este es el caso de una universidad rusa en la que los estudiantes fueron sondeados acerca de la calidad de la educación a distancia y llegaron a conclusiones que merecen una reflexión para la administración universitaria : «Baja auto-organización, falta de control por parte del instructor, falta de interacción efectiva y sensación de aislamiento, lo que obviamente disminuye su satisfacción con la experiencia de aprendizaje en línea» (Markova, Glazkova y Zaborova, 2017: 690). En algunas materias de grados y titulaciones se puede utilizar un aprendizaje mezclado y en línea que resulta, según estudios de metaanálisis revisados en el artículo de Henrie, Bodily, Manwaring y Graham (2015), más satisfactorio y de mayor rendimiento para el estudiante. En el caso de la investigación con una metodología longitudinal intensiva sobre aprendizaje mezclado en un curso de tecnología educativa americano, los autores anteriormente citados hallaron que la claridad instructiva y la relevancia en las actividades influyeron más en la satisfacción de los estudiantes que el medio usado en la instrucción. En otro estudio sobre la materia de Informática Aplicada realizado en una universidad de Checoslovaquia se comparó el resultado de los estudiantes que siguieron el curso de manera presencial y a través de la enseñanza a distancia. Entre las conclusiones del estudio, el autor subrayó aquella que mostraba que los estudiantes de primer curso tenían más dificultad con la enseñanza a distancia (Fojtik, 2015). Dado que son casi seis millones de estudiantes los que hicieron cursos a distancia en 2014, es importante conocer no tanto las matriculaciones y los costes de las mismas, sino determinar las causas del abandono estudiantil y poner remedio a las mismas. El empeño de los investigadores de una universidad norteamericana fue conocer cuando se producía el abandono de los estudiantes que siguieron cursos en línea para mejorar el diseño instructivo, calculando a tal efecto indicadores del momento en que se producía el abandono (Christensen y Spackman, 2017). No cabe duda que un buen diseño instructivo debe incorporar interactividad, calidad global y relevancia y congruencia con las necesidades de los estudiantes. Por ello, la enseñanza en los ambientes en línea deben tener como desafío contante tres constructos interrelacionados: presencia social, presencia docente y presencia cognoscitiva en base a los cuales se pueden formar a un docente universitario. Así ocurrió en el estudio norteamericano de Richardson, Koehler, Besser, Caskurlu, Lim y Mueller (2015) en el que constataron muchos roles docentes de la muestra del estudio durante el proceso de implantación de la enseñanza en línea. Sin duda, el estudio chino de Cheng, Wang, Huang y Zarifis (2016) vino a corroborar que alguna modalidad de colaboración grupal en la enseñanza en línea daba como resultado la satisfacción. Específicamente el concepto de thinklet que es un concepto de colaboración y de evaluación de la usabilidad de la colaboración aplicado al uso de los medios.

\section{Objetivo}

Este estudio presta atención a indicadores académicos que determinan un conocimiento progresivo de la mejora académica en la ULL. Esta investigación analiza medidas asociadas con cinco indicadores de calidad universitaria:

- Revelar la tasa de éxito de los estudiantes, porque reporta una salvaguardia de la excelencia institucional.

Indicadores y control estadístico para el seguimiento y evaluación de preferencias de aprendizaje de estudiantes universitarios. Olga María Alegre de la Rosa y Luis Miguel Villar Angulo. 
- Aquilatar la tasa de eficiencia de los estudiantes para no desperdiciar materiales, tiempo o energía.

- Dilucidar la tasa de graduación de los estudiantes para constatar impedimentos en la culminación de los estudios universitarios.

- Especificar la tasa de abandono universitario para restituir la persistencia estudiantil.

- Explicar la tasa de rendimiento de los estudiantes para aplicar una estrategia de compromiso institucional.

- Desarrollar un análisis DAFO que ilumine factores intervinientes en la planificación y mejora para la toma de decisiones académicas: fortalezas y debilidades internas, y oportunidades y amenazas externas de la ULL.

- $\quad$ Estimular el aprendizaje a distancia a través de medios.

\section{Método}

En el curso académico 2014-2015, la ULL estaba compuesta por 23 centros que impartían licenciaturas y tres centros universitarios asociados, que otorgaron conjuntamente 45 títulos de grado y 64 másteres, además de 52 programas de doctorado y 25 títulos de expertos o especialistas en un total de 25.103 alumnos y alumnas.

Se constituyó una Comisión de 15 miembros expertos (responsables de gestión universitaria y de centros, alumnado y personal de administración y servicios que representaban a la comunidad universitaria de la ULL) (CGE). Esta Comisión, siguiendo la propuesta del documento "U-Ranking (Indicadores Sintéticos del Sistema Universitario Español", que desarrolló el Instituto Valenciano de Investigaciones Económicas (IVIE) (Pérez, Aldás, Aragón y Zaera, 2016) propuso el establecimiento de indicadores sintéticos para facilitar la comparación de los resultados de los estudiantes desagregados por grados y campos de conocimiento mediante el análisis de las tasas de éxito, eficiencia, graduación, abandono y rendimiento, y refirió estrategias alternativas de enseñanza en línea.

El proceso evaluativo de corte cuantitativo consistió en una revisión de indicadores académicos universitarios basado en el estudio de las tasas para cada grado y campo de conocimiento adoptando la especificación numérica de porcentajes anuales de estudiantes según las tasas revisadas de éxito, eficiencia, graduación, abandono y rendimiento:

- Tasa de éxito: relación porcentual entre el número total de créditos superados por los estudiantes matriculados en un curso y el número total de créditos presentados a examen en dicho curso académico (excluidos los créditos adaptados, convalidados, reconocidos, transferidos, etc.).

- Tasa de eficiencia: relación porcentual entre el número total de créditos en los que debieron haberse matriculado los estudiantes graduados de una cohorte de graduación $\mathrm{G}$ para superar un título $\mathrm{T}$ en una Universidad $\mathrm{U}$ y el total de créditos en los que efectivamente se han matriculado los estudiantes graduados de una cohorte de graduación $\mathrm{G}$ en un título T en una Universidad U.

- Tasa de graduación: relación porcentual entre los estudiantes de una cohorte de entrada C que superan, en el tiempo previsto más un año, los créditos conducentes a un título $\mathrm{T}$ en una Universidad $\mathrm{U}$ y el total de los estudiantes de nuevo ingreso de la misma cohorte $\mathrm{C}$ en dicho título $\mathrm{T}$ en la Universidad U.

- Tasa de abandono: relación porcentual entre los estudiantes de una cohorte de entrada C matriculados en el título $\mathrm{T}$ en la Universidad U en el curso académico X, que no se han matriculado en dicho título $\mathrm{T}$ en los cursos $\mathrm{X}+1$ y $\mathrm{X}+2$, y el número total de estudiantes de tal cohorte de entrada $\mathrm{C}$ que accedieron al mencionado título $\mathrm{T}$ el curso académico $\mathrm{X}$.

Indicadores y control estadístico para el seguimiento y evaluación de preferencias de aprendizaje de estudiantes universitarios. Olga María Alegre de la Rosa y Luis Miguel Villar Angulo. 
- Tasa de rendimiento: para un año académico $\mathrm{X}$, relación porcentual entre el número de créditos superados en un título $\mathrm{T}$ en una universidad $\mathrm{U}$ por una cohorte de estudiantes de nuevo ingreso $\mathrm{C} \mathrm{y}$ el número total de créditos matriculados en el título $\mathrm{T}$ en la universidad U por dicha cohorte.

Posteriormente se analizaron las fortalezas, debilidades, oportunidades y amenazas de los grados de centros universitarios, según el esquema DAFO, con el fin de identificar los aspectos positivos y negativos dentro y fuera de las titulaciones. Desde hace años se conoce a DAFO como un marco teórico para la integración de listas de análisis interno y externo en las organizaciones que contribuye a una mejora en la concienciación sobre problemas sociales, económicos o educativos. Aunque DAFO es aparentemente simple puesto que "surgió de la práctica y carecía de fundamento teórico" (Thomas, Chie, Abraham, Raj y Beh, 2014: 115), la búsqueda de la integración analítica por medio de un torbellino de ideas o brainstorming es una meta crítica de cualquier estrategia que pretenda coronar las partes en un todo, desde el aprendizaje personal al organizativo. Los análisis por medio de DAFO se usan ampliamente en sectores industriales relacionados con la ingeniería (Gupta y Mishra, 2016) o académicos para evaluar cambios internos y externos de programas universitarios (Romero-Gutierrez, Jimenez-Liso y Martinez-Chico, 2016; Loureiro, Ferreira, Fresta, Ismail, Rehman y Broome, 2017). Finalmente, incluyeron alternativas de metodología de enseñanza para el aprendizaje mezclado o a distancia, independientemente de los riesgos conocidos que comporta esta estrategia (Markova, Glazkova y Zaborova, 2017).

El procedimiento mantenido por el CGE para ejecutar el análisis DAFO fue el siguiente:

- Contacto con los Coordinadores de las Comisiones Calidad e Innovación Docente de los Centros (CCIDC) de cada uno de los 23 centros de la ULL y uno adscrito, que impartían titulaciones universitarias.

- Utilización de entrevistas, conversaciones, grabaciones, notas de campo en escenarios naturales.

- Manejo de un bloc de papel para representar los razonamientos las distintas Comisiones de Centros por turnos que identificaron y sugirieron las fortalezas que iluminaban y clasificaban los principales puntos fuertes de grados seleccionados. Asimismo procedieron respecto de las debilidades, oportunidades y amenazas de los mismos grados.

- Redacción de un acta con los pasajes estructurales y pragmáticos disponibles de DAFO, que contenía información sobre: día y hora, lugar, asistentes, contenido, mejoras, síntesis y acuerdos.

- Procesamiento de los pasajes observacionales y textos interactivos obtenidos mediante un análisis interpretativo de contenido (Denzin y Lincoln, 2011).

Se ha empleado el paradigma de los métodos mixtos (MMR) (Tashakkori y Teddlie, 2010) como una posibilidad de establecer puente entre enfoques cuantitativos y cualitativos con la posibilidad de combinar las fortalezas de ambos y atenuar sus debilidades. Además, ofrecía la posibilidad de lograr una mejor comprensión del problema que nos ocupa. En esa dirección hemos empleado un diseño propio de los MMR, el incrustado donde uno de los datos se convierte en el soporte del otro.

El elemento cuantitativo se vinculó al lenguaje de los indicadores, estándares o tasas el cual se convirtió para la CGE no solo en expresión de instrumentos prácticos para evaluar el grado en que se integraban los valores y programas de calidad sino también en enunciados dinámicos del pensamiento universitario. A la manera de una base de datos informativa, la antología de definiciones de las tasas sirvió para el control de los resultados de los estudiantes ajustados a los requerimientos de la comunidad universitaria.

Indicadores y control estadístico para el seguimiento y evaluación de preferencias de aprendizaje de estudiantes universitarios. Olga María Alegre de la Rosa y Luis Miguel Villar Angulo. 
La evolución de las tasas de los grados y campos de conocimiento y la comparación de estos entre sí facilitaban un dilatado y ramificado conocimiento de la actividad de enseñanza-aprendizaje de la ULL. El marco DAFO permitió realizar interpretaciones basadas en los datos cuantitativos y analizar las opiniones de las CCIDC sobre los grados de las ramas de conocimiento impartidos en centros universitarios bajo las perspectivas interna (fortalezas y debilidades) y externa (oportunidades y amenazas).

\section{Resultados}

Se muestran los hallazgos de investigación a través de series de representaciones gráficas organizadas por campos de conocimiento, indicadores de éxito, eficiencia, finalización, abandono y logro de estudiantes, y cursos académicos, para posteriormente mostrar el análisis cualitativo llevado a cabo mediante el DAFO de los indicadores obtenidos.

\subsection{Indicadores de resultados universitarios por campos del conocimiento}

La Figura 1 muestra las medias de los porcentajes para los cinco indicadores de tasas por campos científicos. Véase como la tasa de abandono más baja sucede en Ciencias de la Salud y la más alta en Arte y Humanidades. Sin embargo la tasa de éxito es elevada en todos los campos del conocimiento, por encima del $70 \%$ superando en Salud, Ciencias Sociales y Arte y Humanidades el 80\%. Es coherente el dato de eficiencia en los mismos campos científicos por encima del $70 \%$ de eficiencia, al igual que en el rendimiento el cual supera el $50 \%$ en todos los campos, aunque en más bajo en Ingeniería y Arquitectura y el más alto, el de Salud. Por su parte, la graduación resulta algo baja, salvo en Salud que supera el 50\%, no alcanzando ese valor en el resto de campos del conocimiento.

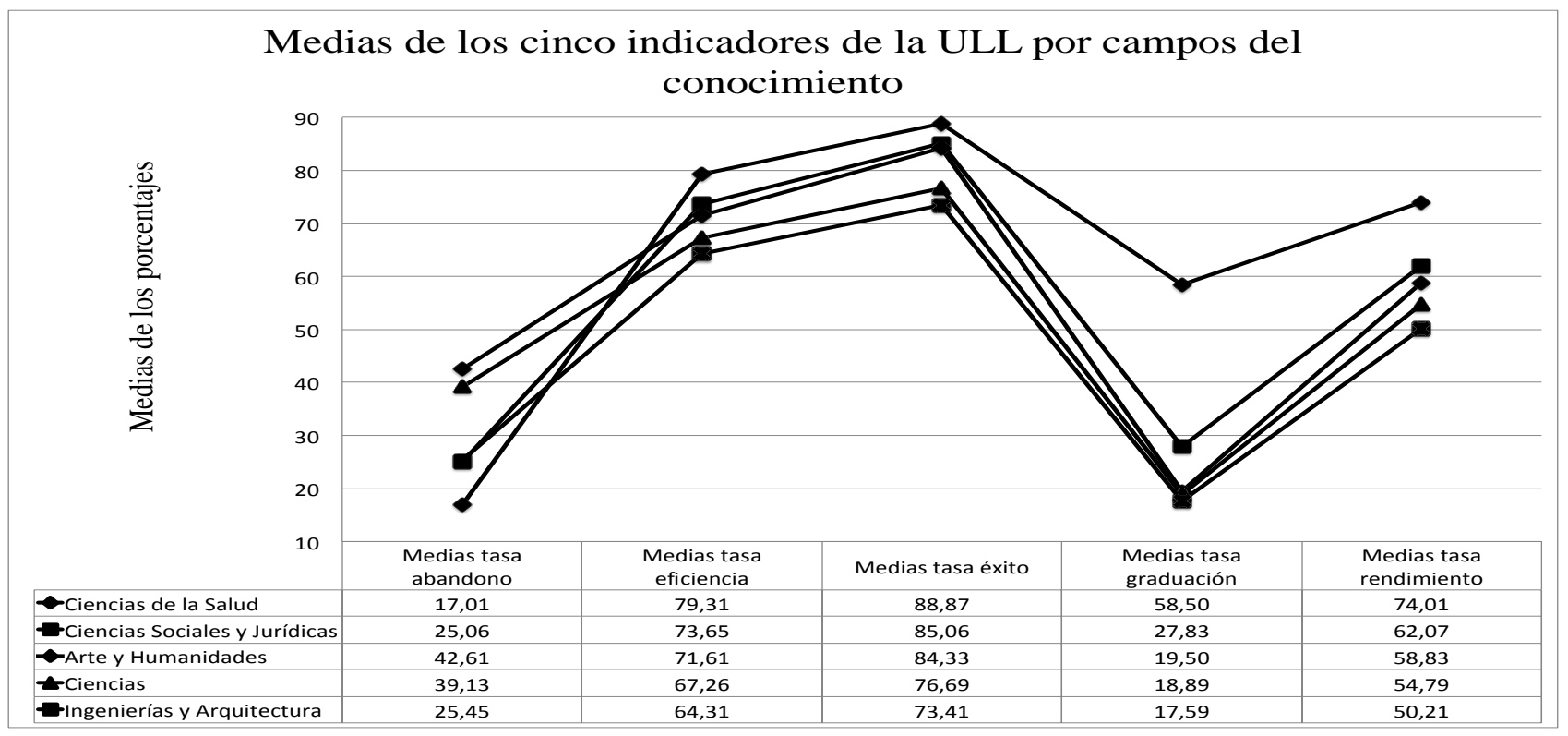

Figura 1. Medias de los porcentajes de los cinco indicadores por campos de conocimiento.

\subsection{Indicadores de tasa de éxito de estudiantes por campos del conocimiento y cursos académicos}

La Figura 2 muestra las medias de los porcentajes de la tasa de éxito de los cinco campos del conocimiento. Ciencias de la Salud incluía seis grados académicos en ocho centros universitarios, y

Indicadores y control estadístico para el seguimiento y evaluación de preferencias de aprendizaje de estudiantes universitarios. Olga María Alegre de la Rosa y Luis Miguel Villar Angulo. 
fue el único campo del conocimiento cuya tasa de éxito mejoró del 2009 al 2012. Los estudiantes que terminaron los cursos de Ciencias de la Salud en el otoño de 2010 fueron el 87,7\% ( $\sigma=1,76)$, mientras que los estudiantes que completaron con éxito el curso en 2012 fueron el 89,94\% $(\sigma=3,85)$. La comparación de los porcentajes en los dos años reveló una mejora en el éxito. En otros términos, los estudiantes universitarios fueron perceptores de éxito en los grados de este campo del conocimiento en dos puntos porcentuales. No obstante, las diferencias intragrado en dicho campo del conocimiento fueron acusadas. Así, mientras los alumnos de grado de Fisioterapia obtuvieron la tasa más alta de éxito $(96,13 \% ; \sigma=0,56)$, los estudiantes de grado de Farmacia consiguieron la tasa media de éxito más baja $(78,16 \% ; \sigma=8,45)$ en el año académico 2011-2012. Por el contrario, Ciencias Sociales y Jurídicas que incluye 15 grados académicos bajó casi diez puntos en cuatro años académicos. Como ejemplo de porcentaje desigual, mientras que el grado de Sociología tuvo la tasa promedio menor $(57,01 \% ; \sigma=8,70)$, el grado de Periodismo obtuvo la tasa media con el porcentaje más alto $(96,41 \% \sigma=1,75)$ de la ULL. Como ejemplo, en 2009-2010 destaca que la tasa media del porcentaje de éxito de los estudiantes para Ciencias Sociales y Jurídicas de la ULL fue 92,68\% ( $\sigma=13,59)$. Aunque la anterior desviación típica es algo elevada para las distintas titulaciones de ese campo del conocimiento, el coeficiente de variación es sólo de 0,16 .

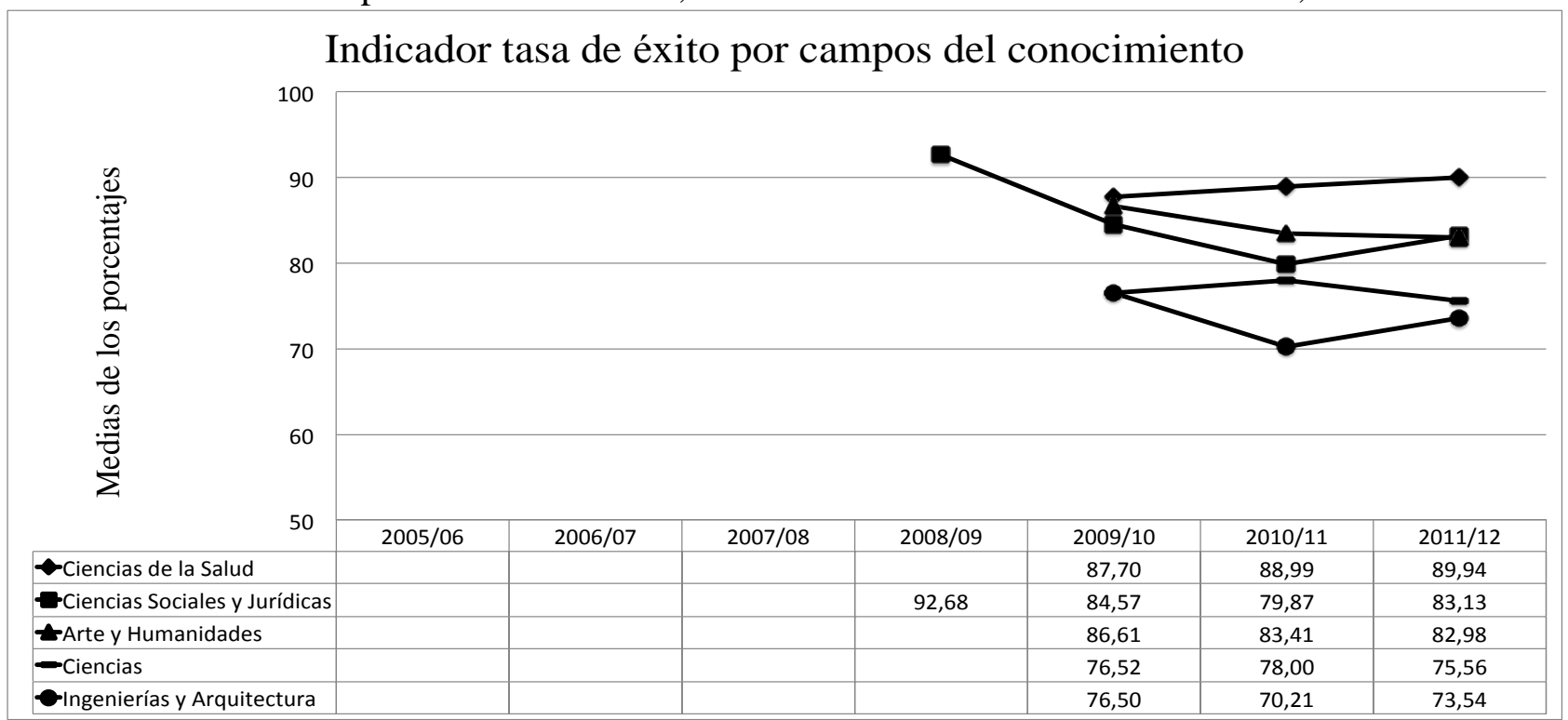

Figura 2. Medias de los porcentajes de la tasa de éxito de estudiantes por campos del conocimiento.

\subsection{Indicadores de tasa de eficiencia de estudiantes por campos del conocimiento y cursos académicos}

Como se mostró en la Figura 1, las medias de porcentajes de la tasa de eficiencia de los cinco campos del conocimiento que no llegan al $80 \%$ de eficiencia sugiere un tipo de patrón de productividad ineficiente. La Figura 3 refleja de manera detallada el indicador de eficiencia por campos del conocimiento y cursos académicos analizados. Ciencias Sociales y Jurídicas e Ingeniería y Arquitectura, que integraba diez grados, muestran mejora en la eficiencia, sobre todo en el curso 2011-2012. El aumento de la tasa de eficiencia de estudiantes que progresaron a través de la finalización de uno de los 14 grados académicos de Ciencias Sociales y Jurídicas fue mayor que 30 puntos (de 63,41\%, $\sigma=13,59$ a 94,50\%, $\sigma=13,59$ ). Las medias de las tasas de este indicador para los grados en Ciencias de la Salud variaron su aspecto entre sí. En este sentido, el grado de Fisioterapia tuvo una tasa promedio del 79,60\% $(\sigma=11,13)$ en $2010-2011$ y la titulación de Farmacia tuvo una tasa promedio de $53,30 \%(\sigma=11,50)$ en el mismo período de tiempo.

Indicadores y control estadístico para el seguimiento y evaluación de preferencias de aprendizaje de estudiantes universitarios. Olga María Alegre de la Rosa y Luis Miguel Villar Angulo. 


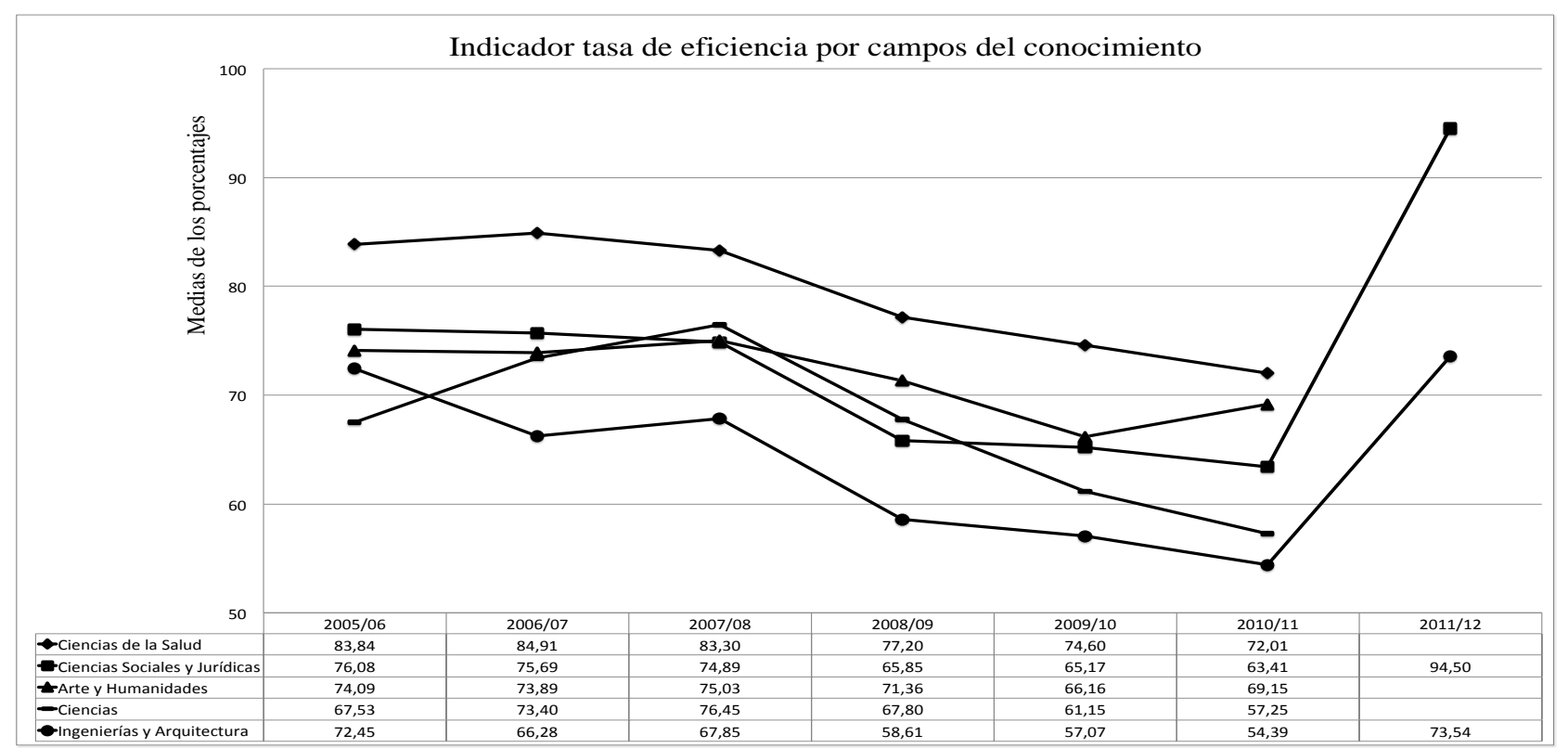

Figura 3. Medias de los porcentajes de la tasa de eficiencia de estudiantes por campos del conocimiento.

\subsection{Indicadores de tasa de graduación de estudiantes por campos del conocimiento y cursos académicos}

Como vimos en la Figura 1, las medias de porcentajes por tasa de graduación, salvo en Ciencias de la Salud, en el resto de campos del conocimiento, está por debajo del 50\%. Las líneas gráficas muestran una idea general subyacente: las tasas de finalización son bajas, pero la tasa de graduación aumentaron cada año del período examinado como muestra la Figura 4. Ciencias de la Salud mejoró la tasa de finalización de los estudiantes muy lentamente, aunque proporcionó la tasa más alta entre todas los campos del conocimiento $(62,44 \% ; \sigma=17,33)$ en 2010-2011. Sin embargo, dentro de este campo del conocimiento, Farmacia tuvo la menor tasa de finalización $(11,30 \% ; \sigma=8,45)$, y Enfermería (Escuela de La Candelaria) la tasa de terminación más alta $(100 \% ; \sigma=0,33)$. Los grados de Ciencias Sociales y Jurídicas mejoraron a lo largo de tiempo: 26,94\% $(\sigma=7,10)$ significó el porcentaje de estudiantes que persiguió uno de los 14 grados en el período 2005-2006, promedio que aumentó a 36,10\% $(\sigma=8,15)$ en cinco años, de 2005-2006 a 2010-2011 (Figura 4). Los estudiantes pertenecientes a al campo de conocimiento de Artes y Humanidades, con ocho grados, fueron los más exitosos en el indicador de graduación finalizando sus títulos en ese período de tiempo: desde un porcentaje medio de 14,73\% (aunque con una elevada desviación según las distintas titulaciones $\sigma=35,65$ ) en el período 2005-2006, a una media en el porcentaje de $25,04 \%$ $(\sigma=17,12)$ en 2010-2011. Mientras tanto, las tasas de terminación en Ingeniería y Arquitectura aumentaron un $9 \%$ en cinco años. De un $14,67 \%(\sigma=7,35)$ en el curso 2005-2006 al 23,74\% $(\sigma=5,10)$ en el 2010-2011.

Indicadores y control estadístico para el seguimiento y evaluación de preferencias de aprendizaje de estudiantes universitarios. Olga María Alegre de la Rosa y Luis Miguel Villar Angulo. 


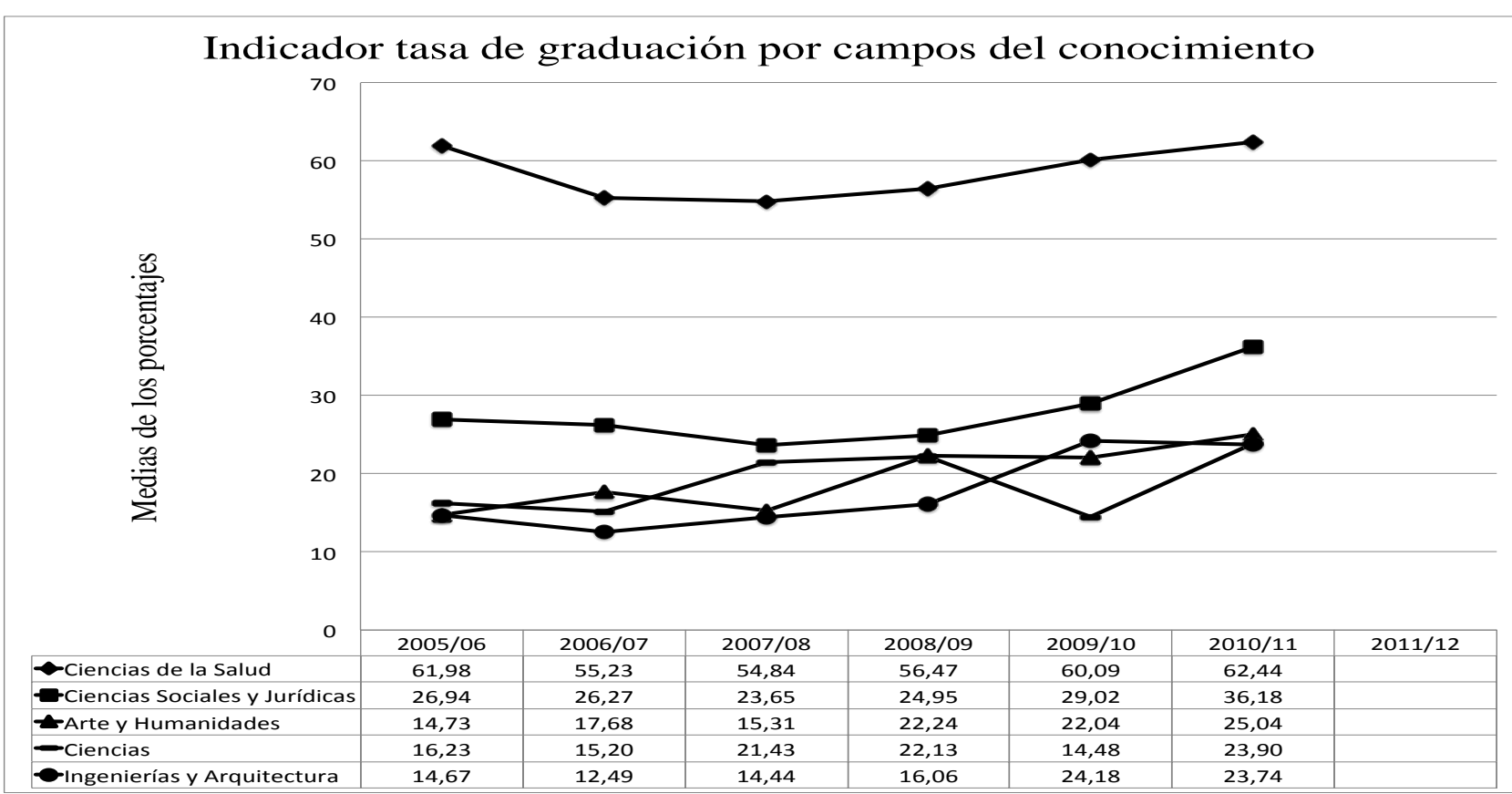

Figura 4. Medias de los porcentajes de la tasa de graduación de estudiantes por campos del conocimiento.

\subsection{Indicadores de tasa de abandono de estudiantes por campos del conocimiento y cursos académicos}

Las medias de los porcentajes de la tasa de abandono en los cinco campos del conocimiento analizados que se muestran en la Figura 5, indican que en los cursos 2009-2010 y 2010-2011 existe una importante reducción en la deserción universitaria. Artes y Humanidades fue el campo del conocimiento que realizó mayores esfuerzos con el fin de reducir la deserción (de 49,95\%; $\sigma=8,05$ en 2005-2006 a 28,20\%; $\sigma=9,66$ en 2010-2011). A pesar de ello, el grado de Filosofía mantuvo la tasa más alta de deserción $(37,80 \% ; \sigma=7,81)$ en 2010-2011, mientras que Filología Española asumió la menor tasa de abandono $(16,90 \% ; \sigma=5,68)$ en el mismo período de tiempo. Siguieron los estudiantes matriculados en Ciencias, con cuatro grados (desde 44,70\%, $\sigma=8,99$ en 2005-2006 a 25,05\%, $\sigma=6,25$ en 2010-2011). El título de Química conservó la tasa de deserción más alta $(31,60 \% ; \sigma=9,99)$, mientras que Biología poseyó la tasa menor de abandono $(17,90 \% ; \sigma=12,35)$ en el mismo año académico 2010-2011. Ciencias de la Salud conservó aproximadamente la misma tasa de retención de los estudiantes a lo largo de los seis años. En este campo del conocimiento, el grado de Medicina disfrutó la menor tasa de abandono $(5,40 \% ; \sigma=7,75)$, mientras que Logopedia adquirió la tasa más alta $(23,90 \% ; \sigma=5,10)$. Ingeniería y Arquitectura revelaron que estaban reduciendo lentamente la deserción universitaria en 2010-2011 (de 27,10\%; $\sigma=13,99$ en 2005-2006 a 25,60\%; $\sigma=14,86$ en $2010-2011)$.

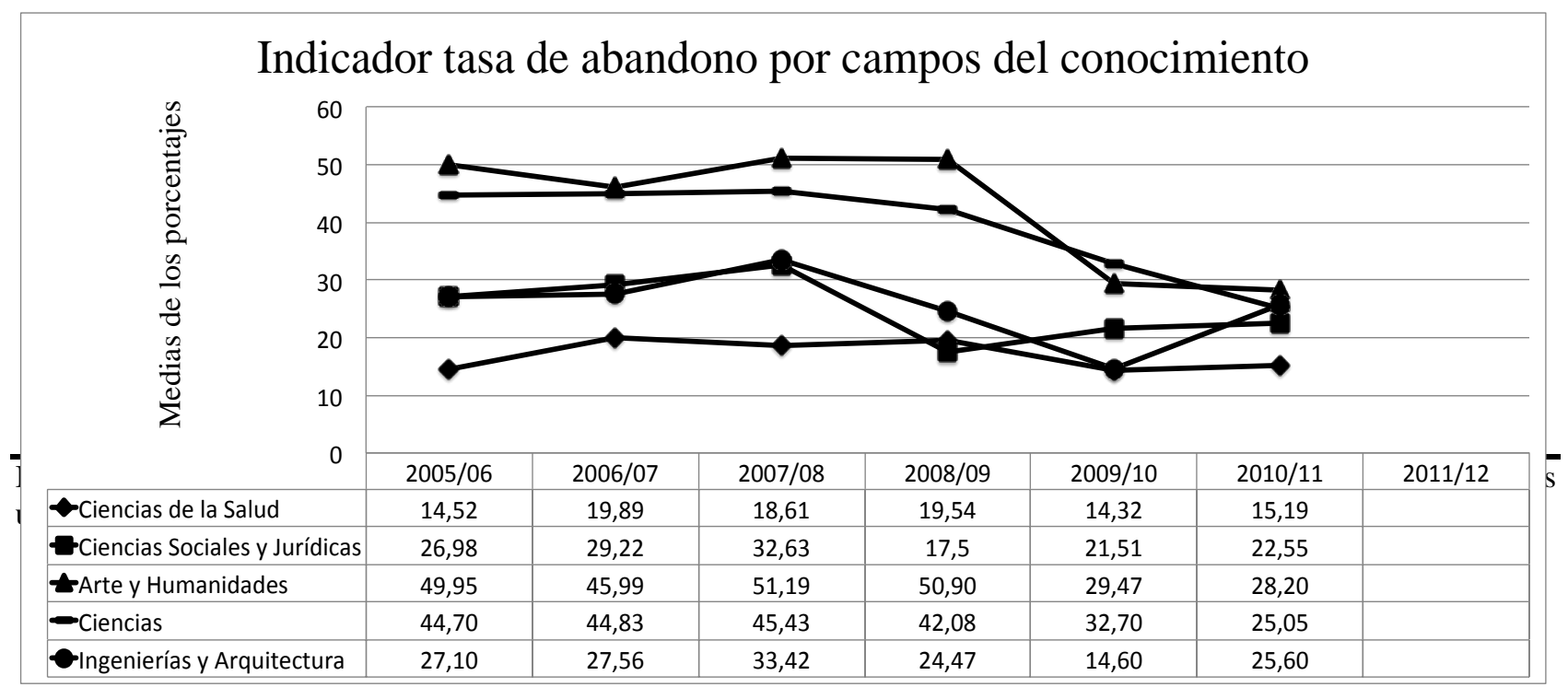


Figura 5. Medias de los porcentajes de la tasa de abandono de estudiantes por campos del conocimiento.

\subsection{Indicadores de tasa de rendimiento de estudiantes por campos del conocimiento y cursos académicos}

La Figura 6 muestra por campos del conocimiento el indicador tasa de rendimiento de los estudiantes de la ULL por curso académico. Se destaca que Ciencias Sociales y Jurídicas reveló una tasa media mejorada de casi diez puntos en seis años (desde 55,95\%; $\sigma=11,99$ en 2005-2006 a 65,02\%; $\sigma=9,18$ en el año académico 2011-2012). El grado de Periodismo gozó de la tasa media más alta $(87,70 ; \sigma=5,32)$ en el año académico 2011-2012 y el grado de Sociología la tasa de logro más baja $(46,00 \% ; \sigma=7,99)$ en el mismo período de tiempo. Mientras que a nivel nacional la tasa media de esta rama de conocimiento fue $68,82 \%$ en el curso 2010-2011. Durante este tiempo, los grados de Ciencias de la Salud revelaron un esfuerzo continuo y ascendente de logro. Los estudiantes mejoraron la tasa promedio para obtener el título (de 70,00\%; $\sigma=11,99$ en 2005-2006 a $79,81 \% ; \sigma=9,26$ en 2011-2012). El grado de Enfermería tuvo la mayor tasa de rendimiento $(89,30 \% ; \sigma=6,01)$ en el período 2011-2012, y la titulación de Farmacia, la tasa más baja $(61,40 \%$; $\sigma=11,23)$ en el mismo período de tiempo. Simultáneamente, la tasa promedio nacional de Ciencias de la Salud fue 79,63\% en 2010-2011. La Figura 6 detalla que la rama de Ingenierías y Arquitectura, mejora un punto su tasa media en seis años siendo la titulación de Ingeniería Civil la que obtuvo la tasa media más alta de rendimiento $(59,30 \% ; \sigma=13,99)$ en el período 2011-2012, mientras que el grado de Ingeniería Radioelectrónica tuvo la tasa promedio más baja de logro $(31,30 \% ; \sigma=8,72)$ en el mismo año académico.

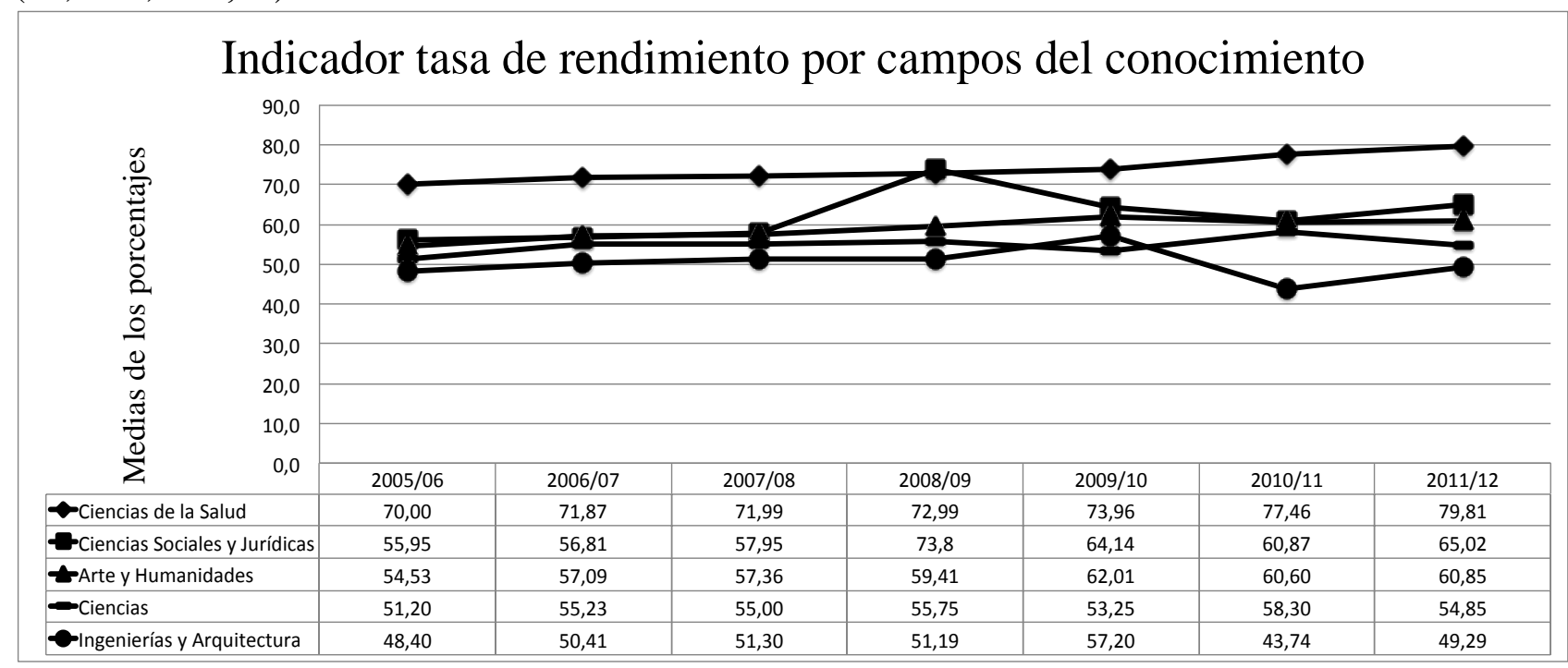

Figura 6. Medias de los porcentajes de la tasa de rendimiento de estudiantes por campos del conocimiento.

\subsection{Análisis DAFO de las percepciones de las Comisiones de Calidad de los diferentes Grados de la ULL}

Se utilizó un análisis de contenido interpretativo de datos textuales y pragmáticos (Denzin y Lincoln, 2011) proyectando las percepciones y conocimientos conceptuales de las CCIDC en una matriz dos por dos (Tabla 1) que contiene de manera general las Fortalezas y Oportunidades obtenidas a partir de la opinión de dichas comisiones al analizar los datos de los Indicadores anteriormente presentados (Thomas, Chie, Abraham, Raj y Beh, 2014).

Indicadores y control estadístico para el seguimiento y evaluación de preferencias de aprendizaje de estudiantes universitarios. Olga María Alegre de la Rosa y Luis Miguel Villar Angulo. 
Destacamos entre las fortalezas la satisfacción con los programas formativos, mientras que las oportunidades se refieren a la demanda de ingreso en ciertas titulaciones. El argumento más destacado dirigido al profesorado se reseñó para la docencia y su calidad como siguen revelando estudios como el de Casero-Martínez (2016), al tiempo que las oportunidades subrayaban el reconocimiento social a la innovación tecnológica e idiomas modernos. Las CCIDC han analizado los indicadores y destacan la disminución de las tasas de abandono, dando más oportunidades a la demanda empresarial de perfiles multidisciplinares. También han destacado la fortaleza de las actividades prácticas y extracurriculares y las oportunidades para el desarrollo de alianzas estratégicas internacionales y de excelencia en la investigación.

Tabla 1. Matriz del análisis DAFO para las Fortalezas y Oportunidades.

\begin{tabular}{|c|c|}
\hline Fortalezas (F-) & Oportunidades (O-) \\
\hline $\begin{array}{l}\text { F1. Los resultados académicos de las titulaciones de grado son } \\
\text { mejores que los observados en las licenciaturas, diplomaturas o } \\
\text { ingenierías. } \\
\text { F2. La evaluación continua mejora los resultados de aprendizaje } \\
\text { del alumnado. } \\
\text { F3. Se han reducido las tasas de abandono en la mayor parte de } \\
\text { las titulaciones. } \\
\text { F4. Los centros se han dotado de instrumentos y estructuras } \\
\text { orientadas a la mejora y el aseguramiento de la calidad. } \\
\text { F5. Las guías docentes de las asignaturas se presentan como } \\
\text { herramientas en el desarrollo de las actividades docentes. } \\
\text { F6. Aumento generalizado sobre la importancia de la docencia y } \\
\text { de su calidad por parte de las CCIDC de los centros. } \\
\text { F7. Sensibilización creciente por parte de los equipos directivos } \\
\text { de los centros y del profesorado en relación con el desarrollo y } \\
\text { la calidad de los programas formativos. } \\
\text { F8. Toma de conciencia creciente por parte de la comunidad de } \\
\text { cada centro con relación a los procesos de acreditación. } \\
\text { F9. Alto grado de satisfacción del alumnado con el desarrollo de } \\
\text { algunos programas formativos. } \\
\text { F10. Amplia oferta de actividades extracurriculares en los } \\
\text { centros: congresos, jornadas, talleres, cursos, etc. } \\
\text { F11. Establecimiento de convenios y acuerdos para el } \\
\text { establecimiento de las titulaciones de la ULL en sedes de otras } \\
\text { islas/municipios } \\
\text { F12. Mayor conexión de los grados y másteres con la realidad } \\
\text { social, con las empresas y el mercado laboral. } \\
\text { F13. La evaluación continua y los planes de tutorías han sido un } \\
\text { punto fuerte para vehicular la mejor comunicación profesor- } \\
\text { alumno y la mejor valoración del trabajo de cada uno. } \\
\text { F14. La ULL cuenta con un campus de excelencia internacional. }\end{array}$ & $\begin{array}{l}\text { O1. El interés de instituciones públicas de otros municipios o } \\
\text { islas en contar en su territorio con estudios de educación } \\
\text { superior. } \\
\text { O2. Utilización del campus de excelencia e internacionalización } \\
\text { como medio para ampliar el mercado potencial de alumnos/as. } \\
\text { O3. Aumento de la demanda de titulaciones oficiales en } \\
\text { modalidad semipresencial y/o enseñanza a distancia como nicho } \\
\text { para captar alumnado de otras regiones y países, principalmente } \\
\text { hispano hablantes, desplegando y potenciando la utilización del } \\
\text { campus virtual de la ULL. } \\
\text { O4. Elevada demanda de alumnado de nuevo ingreso en algunas } \\
\text { titulaciones } \\
\text { O5. La colaboración del tejido empresarial con la universidad, } \\
\text { para el desarrollo de la asignatura de prácticas externas. } \\
\text { O6. Sistema educativo homologable en cualquier país de la } \\
\text { Unión Europea. } \\
\text { O7. Alto reconocimiento social a la innovación docente en el } \\
\text { ámbito tecnológico, uso del idioma y de prácticas externas. } \\
\text { O8. Mayor demanda de perfiles multidisciplinares por parte de } \\
\text { las empresas. } \\
\text { O9. Mayor consenso social de la necesidad de un cambio de } \\
\text { modelo socioeconómico en Canarias que potencia el } \\
\text { conocimiento como valor añadido. } \\
\text { O10. Europa } 2020 \text { y Especialización Inteligente RIS3 son } \\
\text { políticas europeas que se centran en la I+D+i como motor de } \\
\text { salida de la crisis. } \\
\text { O11. Crecimiento en Latino América y África que abren nuevos } \\
\text { mercados laborales para nuestros titulados. } \\
\text { O12. Aumento del valor social del emprendimiento, que mira a } \\
\text { la Universidad como centro principal de generación de } \\
\text { proyectos. }\end{array}$ \\
\hline
\end{tabular}

Se presenta en la Tabla 2 el resumen con las dimensiones analizadas por las CCIDC de cada Centro. Para las Debilidades y Amenazas, se detallan las opiniones relativas al programa formativo (asignaturas ubicadas inadecuadamente en el plan de estudios, grupos con excesivo número de alumnado con amenazas relativas a la poca oferta de titulaciones online de la ULL), recursos e infraestructuras (claras deficiencias en infraestructuras y apoyo económico a la universidad), alumnado (con manifiestas dificultades en el acceso y elección de carrera y amenazas vinculadas a la escasez de becas y la limitada orientación previa a la universidad), profesorado (claras dificultades formativas o de coordinación) o a la dimensión institucional (fundamentalmente vinculada a faltas de implicación y liderazgo y escaso reconocimiento).

Tabla 2. Matriz del análisis DAFO para las Debilidades y Amenazas según dimensiones.

Indicadores y control estadístico para el seguimiento y evaluación de preferencias de aprendizaje de estudiantes universitarios. Olga María Alegre de la Rosa y Luis Miguel Villar Angulo. 


\begin{tabular}{|c|c|}
\hline \multicolumn{2}{|c|}{ Dimensión Programa Formativo (-F) } \\
\hline Debilidades (D-) & Amenazadas (A-) \\
\hline $\begin{array}{l}\text { DF1. Se detectan asignaturas ubicadas inadecuadamente en el } \\
\text { plan de estudios } \\
\text { DF2. Hay contenidos de las asignaturas de formación básica que } \\
\text { no se ajuntan adecuadamente al perfil de la titulación } \\
\text { DF3. Se detectan asignaturas que, de manera continuada, } \\
\text { presentan un número elevado de suspensos (tasas bajas de } \\
\text { eficiencia) o no presentados (tasas bajas de éxito). } \\
\text { DF4. No cumplimiento de los resultados y objetivos previstos } \\
\text { en la Memoria de Verificación del Título. } \\
\text { DF5. Grupos con excesivo número de alumnado. } \\
\text { DF6. El esfuerzo dedicado a la formación básica en el primer } \\
\text { curso no está dando los resultados esperados de movilidad entre } \\
\text { titulaciones de la misma rama. } \\
\text { DF7. La asignación de créditos en algunas asignaturas del plan } \\
\text { de estudios no se corresponde con las exigencias y cargas de } \\
\text { trabajo a realizar para su superación. } \\
\text { DF8. Inexistencia de un sistema de evaluación homogéneo a } \\
\text { nivel título. } \\
\text { DF9. En algunos casos, el perfil de egreso previsto no se } \\
\text { corresponde con el perfil de egreso real. } \\
\text { DF10. El diseño e implantación de algunos títulos no favorece } \\
\text { el proceso de enseñanza-aprendizaje centrado en el estudiante. }\end{array}$ & $\begin{array}{l}\text { AF1. Existencia de una competencia cada vez mayor entre } \\
\text { universidades nacionales e internacionales, públicas y privadas, } \\
\text { respecto a una oferta formativa más atractiva, con mejores } \\
\text { resultados, y ajustada a los intereses del alumnado. } \\
\text { AF2. Aumento de la oferta de titulaciones oficiales en } \\
\text { modalidad semipresencial u online adaptadas a los nuevos } \\
\text { escenarios formativos demandados por la sociedad, y que están } \\
\text { desarrolladas de manera insuficiente en la ULL. } \\
\text { AF3. Poco conocimiento social de los modelos educativos } \\
\text { extranjeros (europeo y americano) que nos asemejan con el } \\
\text { sistema de Bolonia a ellos. } \\
\text { AF4. Poco valor social y universitario de la oferta de posgrado } \\
\text { como vía de mejora para el acceso al mercado laboral. }\end{array}$ \\
\hline
\end{tabular}

Dimensión Recursos e Infraestructuras (-R)

DR1. Imposibilidad material y logística de responder a la planificación prevista para el desarrollo de título.

DR2. En algunos centros las infraestructuras (aulas y laboratorios) y los recursos humanos y de apoyo a la docencia con los que se cuenta imposibilitan la configuración de grupos de alumnos/as más reducidos (teóricos y prácticos) para lograr una docencia de mayor calidad.

DR3. En algunas titulaciones hay carencias de personal académico con los niveles de dedicación requeridos.
AR1. La financiación actual con la que cuenta la ULL y la falta de un sistema de financiación estable en el tiempo impide una planificación de recursos humanos y materiales a medio y a largo plazo

AR2. Existencia de normativas nacionales de obligado cumplimiento que limitan las posibilidades de contratación y promoción del personal académico.

AR3. Caída paulatina de la inversión en la educación superior que limita o reduce la innovación en el ámbito docente.

Dimensión Alumnado (-A)

DA1. El tipo de Pruebas de Acceso a la Universidad.

DA2. En algunas titulaciones el alumnado que accede no las elije en primera opción.

DA3. Escasa utilización de la tutoría académica y de carrera por parte del alumnado, que les lleva a una toma de decisiones académico-profesionales inadecuadas por falta de asesoramiento.
AA1. Aumento del número de alumnos que eligen otras universidades para realizar estudios superiores.

AA2. El déficit de orientación en la formación secundaria inmediatamente anterior al acceso a la Universidad.

AA3. El alumnado de nuevo ingreso accede a los estudios universitarios con déficits formativos.

AA4. La crisis económica ha reducido la capacidad de financiación por parte de las familias, así como por la reducción del sistema de becas.

AA5. Pérdida de confianza en el valor de un título universitario para encontrar empleo.

AA6. Rechazo a la movilidad lo que influye en la selección de itinerarios académicos y profesionales.

AA7. Nuevos modelos educativos de secundaria que no tienen continuidad en la Universidad.

AA8. Impacto negativo en la sociedad de la información sobre los ranking universitarios donde no se refleja, ni valora la calidad docente.

Dimensión Profesorado (-P)

DP1. Déficit de coordinación docente, tanto horizontal, entre profesores de la misma asignatura y curso, como vertical, entre profesores de asignaturas de cursos diferentes.

DP2. En sectores importantes del profesorado aún no se ha
AP1. Pérdida de profesorado que ve en otras universidades/ instituciones/ corporaciones... ofertas profesionales más atractivas (académicas, investigadoras, etc.) AP2. Cultura de rechazo a los cambios por parte de algunos

Indicadores y control estadístico para el seguimiento y evaluación de preferencias de aprendizaje de estudiantes universitarios. Olga María Alegre de la Rosa y Luis Miguel Villar Angulo. 
producido la necesaria adaptación al modelo de enseñanzaaprendizaje propios del EEES.

DP3. En ocasiones el profesorado no consigue que los alumnos adquieran las competencias asociadas a cada asignatura/curso.

DP4. La carga de trabajo del alumno excede con frecuencia lo establecido en la guía docente de la asignatura.

DP5. Heterogeneidad en los criterios y sistemas de evaluación y las formas de aplicación de los mismos.

DP6. Imposibilidad de desarrollar la actividad docente de forma adecuada debido al exceso de carga docente.

DP7. Escasa implicación del profesorado en los procesos de gestión académica. profesores.

AP3. Débil reconocimiento de la importancia de la formación en competencias frente a la clásica formación basada únicamente en el currículum

AP4. Altas capacidades tecnológicas y lingüística en el segundo idioma por parte del alumnado que limita la iniciativa de muchos profesores que no ha adquirido tal nivel de competencia en las mismas.

Dimensión Institucional (-I)

DI1. Incumplimiento de las directrices docentes establecidas por los centros y ausencia de respaldo institucional.

DI2. Falta del reconocimiento a la labor del profesorado.

DI3. Excesivo número de convocatorias y acumulación de exámenes en corto espacio de tiempo.

DI4. La aplicación de la normativa de permanencia puede ocasionar aumento de la tasa de abandono.

DI5. Falta de adecuación de los procesos de gestión académica y administrativa para responder a los procesos de acreditación.

DI6. Falta de implicación por parte de las direcciones de los departamentos en el desarrollo de los programas formativos.

DI7. Inexistencia de mecanismos de seguimiento y supervisión de la actividad docente por parte de los departamentos.

DI8. Exceso de carga docente en determinadas áreas de conocimiento y departamentos.

DI9. Sobrecarga de trabajo docente de titulaciones debido al alto número de asignaturas y/o grupos de alumnos.

DI10. Asignaturas con excesivo número de profesores que dificultan el desarrollo de la misma.
AI1. Pérdida de alumnado por el incremento de tasas universitarias.

AI2. Reformas universitarias cambiantes.

AI3. Falta de estabilidad en el marco nacional de la enseñanza superior.

AI4. Necesidad de cumplir con las exigencias de evaluación y calidad establecidas por el MEC y las agencias nacionales y autonómicas para garantizar la continuidad o no de una titulación.

AI5. Transparencia institucional y rendición de cuentas a la sociedad que permite a los grupos de interés comparar las ofertas de enseñanza superior.

AI6. Débil o nulo sistema público de revisión y evaluación de la calidad docente en la educación superior.

AI7. Falta de concienciación sobre los procesos de acreditación de las titulaciones universitarias.

\section{Discusión}

Primero, los resultados han demostrado que el campo de Ciencias de la Salud fue el único que mejoró las tasas de éxito a lo largo de los años analizados. Parece que la satisfacción de los estudiantes universitarios con los títulos fue algo importante, ya que contribuye al éxito de los estudiantes, sobre todo, observado en el grado de Fisioterapia. En este punto, se llevaron a cabo cambios curriculares significativos en varios países, mientras los estudiantes exigen un camino de transición en la titulación profesional: del grado de máster al de doctorado, como ya había ocurrido fuera de España (Rapport, Stelzner y Rodríguez, 2007). Esto significa que los estudiantes de Fisioterapia persistieron en su compromiso de aprendizaje de nuevos cursos y grados académicos en la ULL.

Segundo, las tasas de deserción de los estudiantes universitarios de la rama de Ingeniería y Arquitectura continúan siendo un motivo de preocupación, que implica rediseñar la práctica curricular, en particular la relación entre las características de entrada de los estudiantes a la titulación y de integración académica y las medidas de resultados académicos de Ingeniería (Marra, Tsai, Bogue y Pytel, 2015) para lograr que esta rama de conocimiento sea eficiente. Los datos aportados por Villar-Aguilés, Hernández y Dobon y García-Ros (2017) sobre los abandonos de estudios universitarios mediante el uso del concepto de "reubicación académica" para analizar

Indicadores y control estadístico para el seguimiento y evaluación de preferencias de aprendizaje de estudiantes universitarios. Olga María Alegre de la Rosa y Luis Miguel Villar Angulo. 
trayectorias diversas durante seis cursos académicos en los que constatan que diferencias apreciadas entre titulaciones y su agrupación por ramas de conocimiento permiten constatar la importante heterogeneidad institucional existente. Christensen y Spackman (2017) informan de la complejidad conceptual para definir el abandono y proponen, en el caso de la enseñanza en línea, distintos momentos en los que se podría detener el abandono a través de tabiques de contención del curso.

Tercero, las tasas de graduación en los grados duraron desde cinco años en Farmacia $(11,30 \%)$ a cuatro años en Enfermería (100\%) en el campo de Ciencias de la Salud. De gran preocupación puede parecer la extremadamente baja tasa de finalización de la población del campo Artes y Humanidades, en particular en el grado de Historia del Arte $(8,10 \%)$, aunque los estudiantes matriculados en este campo del conocimiento fueron aumentando la tasa de graduación en 20102011. Además, el campo de Ingeniería y Arquitectura fue aumentando el porcentaje de titulados, aunque el número de graduados aún se consideró baja. Si la ULL tiene que cambiar la tendencia en este campo del conocimiento debería seguir una de las dos rutas sugeridas por Morris (2012: 168): "Aumentar las matrículas o más graduaciones".

Cuarto, según lo aconsejado por Donhardt (2013: 207), "el desgaste del primer año de pregrado representa la mayor pérdida en números absolutos de estudiantes entrantes." Por desgracia, los datos sobre rendimiento académico en el primer año de los estudiantes de los campos de conocimiento no están disponibles. El grado de Filosofía tuvo la tasa de deserción más alta (37,80\%) en 2010-2011. En consecuencia, el campo de Artes y Humanidades manifestó el porcentaje de deserción más alto entre todos los campos del conocimiento (28,20\% en 2010-2011). Para invertir esta tendencia de abandono, la ULL tendría que adoptar una mayor determinación para detectar fracasos tempranos y proporcionar apoyo a los estudiantes que estuvieran en riesgo de borrarse de clase.

Quinto, los grados del campo Ciencias de la Salud mostraron un esfuerzo continuo y ascendente de logro. En paralelo a ella, el campo de Ciencias Sociales y Jurídicas reveló una tasa media mejorada de casi diez puntos en seis años, mientras que el campo de Ingeniería y Arquitectura mejoró sólo un punto su tasa promedio en el mismo período. Los grados opuestos fueron Enfermería, que tuvo la mayor tasa de logro $(89,30 \%)$ en el período 2011-2012, y en la otra cara de la moneda Ingeniería Radioelectrónica que obtuvo la tasa promedio más baja $(31,30 \%)$ en el mismo periodo de tiempo. Sexto, las medidas propuestas por la CGE han ido en consonancia con el "Protocolo de Renovación de la Acreditación de la Red Española de Agencias de la Calidad Universitaria". Entre otras medidas se plantearon las siguientes: "Establecimiento de Pruebas de Acceso específicas para cada titulación o para los títulos de las distintas ramas de conocimientos", "Seguimiento y revisión de los métodos docentes desplegados en las asignaturas con tasas elevadas de suspenso, abandono y éxito", "Reducir el número de alumnado por grupo".

Finalmente, séptimo, es posible que la ULL defina un plan estructurado de mejora para el aprendizaje y el rendimiento de los estudiantes, como un curso cero para cada titulación o el más popular "Programa de Experiencia de Primer Año", que está en línea con la opinión de Nadelson, Semmelroth, Martínez, Featherstone, Fuhriman y Vender (2013), que mezclaba las expectativas y las decisiones de los estudiantes para asistir a la universidad en el primer curso universitario. De igual modo se precisa una revisión de las estrategias de información y orientación al alumnado preuniversitario y dirigir las actividades de los planes de tutoría universitaria hacia las competencias transversales en las que se haya detectado mayor déficit entre el alumnado de cada titulación.

Indicadores y control estadístico para el seguimiento y evaluación de preferencias de aprendizaje de estudiantes universitarios. Olga María Alegre de la Rosa y Luis Miguel Villar Angulo. 
Se propone además, la mejora en la coordinación docente en entornos virtuales dentro de cada asignatura y mayor seguimiento y evaluación de los métodos docentes y aprendizajes colaborativos en línea con el establecimiento de cursos de formación docente para el profesorado universitario en la dirección que Alegre y Villar (2007) manifestaron en su estudio sobre cinco universidades españolas, o sobre ámbitos de interacción docente en cursos de formación en línea (Villar y Alegre, 2008) con el desarrollo de modelos que impliquen la presencia del instructor en el aprendizaje en línea, el liderazgo distribuido y la implicación de estudiantes según contextos ambientales.

\section{Conclusiones}

La CGE ha capturado la información esencial que guía el aseguramiento de la calidad nuclear y la mejora de los grados y campos de conocimiento en la ULL, mediante:

- Seguimiento y revisión de la metodología docente con la incorporación del aprendizaje mezclado y colaborativo en línea de asignaturas de grados y campos de conocimiento con tasas elevadas de suspenso, abandono y éxito.

- Revisión de indicadores y ajuste de tasas detalladas en cinco campos de conocimiento para cultivar una transformación institucional, según el sentido de excelencia universitaria propuesto por los miembros de la CGE.

- Análisis DAFO de percepciones de los miembros de los CCIDC para calificar seis temas institucionales que pueden modernizar y profesionalizar la ULL dentro de una Educación Superior diversificada, según manifiesta la misión de EURASHE (Asociación Europea de Instituciones de Educación Superior) de 2015.

Presentación del artículo: 12 de octubre de 2017

Fecha de aprobación: 3 de diciembre de 2017

Fecha de publicación: 22 de diciembre de 2017

Alegre de la Rosa, O.M. y Villar Angulo, L.M. (2017). Indicadores y control estadístico para el seguimiento y evaluación de preferencias de aprendizaje de estudiantes universitarios. RED. Revista de Educación a Distancia, 55. Consultado el (dd/mm/aaaa) en http://www.um.es/ead/red/55

\section{Financiación}

Esta investigación no ha recibido ninguna subvención específica de los organismos de financiación en los sectores públicos, comerciales o sin fines de lucro.

\section{Referencias}

Al-Arimi, A.M.A.-K. (2014). Distance Learning. Procedia - Social and Behavioral Sciences, 152, 82-88.

Alegre, O.M., \& Villar, L.M. (2007). Evaluación de la formación en línea del profesorado de cinco universidades españolas. Revista de Universidad y Sociedad del Conocimiento (RUSC), 1(4), 1-13.

Indicadores y control estadístico para el seguimiento y evaluación de preferencias de aprendizaje de estudiantes universitarios. Olga María Alegre de la Rosa y Luis Miguel Villar Angulo. 
Arce, M.E, Crespo, B., \& Míguez-Álvarez, C. (2015). Higher Education Drop-Out in SpainParticular Case of Universities in Galicia. International Education Studies, 8(5), 247-264. http://dx.doi.org/10.5539/ies.v8n5p247.

Bowman, N.A. (2014). Conceptualizing Openness to Diversity and Challenge: Its Relation to College Experiences, Achievement, and Retention. Innovative Higher Education, 39(4), 277-291. https://doi.org/10.1007/s10755-014-9281-8.

Burd, E., \& Hodgson, B. (2016). Attendance and Attainment: a five year study. Innovation in Teaching and Learning in Information and Computer Sciences, 5(2), 1-12.

Casero-Martínez, A. (2016). Deconstrucción del "buen profesor". Análisis de las características formales $\mathrm{y}$ no formales de la docencia universitaria. RELIEVE, 22(2), art. 4. http://dx.doi.org/10.7203/relieve.22.2.9419.

Çelik, E. (2015). Mediating and moderating role of academic self-efficacy in the relationship between student academic support and personal growth initiative. Australian Journal of Career Development, 24(2), 105-113. https://doi.org/10.1177/1038416215583563.

Cheng, X., Wang, X., Huang, J., \& Zarifis, A. (2016). An Experimental Study of Satisfaction Response: Evaluation of Online Collaborative Learning. International Review of Research in Open and Distributed Learning, 17(1), January, 60-78. http://dx.doi.org/10.19173/irrodl.v17i1.2110.

Christensen, S.S., \& Spackman, J.S. (2017). Dropout Rates, Student Momentum, And Course Walls: A New Tool For Distance Education Designers. Journal of Educators Online, 14(2). https://www.thejeo.com/archive/archive/2017_142/christensen_spackmanpdf.

Contreras, F., \& Contreras, G.J. (2015). Raising the Bar for Hispanic Serving Institutions: An Analysis of College Completion and Success Rates. Journal of Hispanic Higher Education, 14(2), 151-170. https://doi.org/10.1177/1538192715572892.

Denzin, N.K., \& Lincoln. Y.S. (2011). The Sage Handbook of Qualitative Research. Fourth Edition. Thousand Oaks: Sage Publications, Inc.

Donhardt, G.L. (2013). The Fourth-Year Experience: Impediments to Degree Completion. Innovative Higher Education, 38(3), 207-221.

Dumford, A.D., \& Miller, A.L. (2015). Assessing alumni success: income is NOT the only outcome! Assessment \& Evaluation in Higher Education, 42(2), 195-207. http://dx.doi.org/10.1080/02602938.2015.1098587.

El Hassan, K. (2014). Investigating Conditions for Student Success at an American University in the Middle East. Higher Education Studies, 4(5), 62-74. Doi:10.5539/hes.v4n5p62.

Elliott, D.C. (2016). The impact of self-beliefs on post-secondary transitions: The moderating effects of institutional selectivity. High Education, 71(3), 415-431.

EURASHE (Asociación Europea de Instituciones de Educación Superior) (2015). Recuperado de https://www.eurashe.eu/library/151020-23-redue-buenos-aires-bmr_ppt-pdf/ $\quad(10$ mayo

Indicadores y control estadístico para el seguimiento y evaluación de preferencias de aprendizaje de estudiantes universitarios. Olga María Alegre de la Rosa y Luis Miguel Villar Angulo. 
2017).

Fojtik, R. (2015). Comparison of full-time and distance learning. Procedia - Social and Behavioral Sciences, 182, 402-407.

Ghanizadeh, A. (2016). The interplay between reflective thinking, critical thinking, self-monitoring, and academic achievement in higher education. Higher Education, 74(1), 1-14. Doi: 10.1007/s10734-016-0031-y.

Gupta, G., \& Mishra, R.P. (2016). A SWOT analysis of reliability centered maintenance framework. Journal of Quality in Maintenance Engineering, 22(2), 130-145.

Hen, M., \& Goroshit, M. (2014). Academic Procrastination, Emotional Intelligence, Academic SelfEfficacy, and GPA: A Comparison Between Students With and Without Learning Disabilities. Journal of Learning Disabilities, 47(2), 116-124 . Doi: $10.1177 / 002219412439325$.

Henrie, C.R., Bodily, R., Manwaring, K.C., \& Graham, Ch. R. (2015). Exploring Intensive Longitudinal Measures of Student Engagement in Blended Learning. International Review of Research in Open and Distributed Learning, 16(3), 131-155. http://dx.doi.org/10.19173/irrodl.v16i3.2015.

Heublein, U. (2014). Student drop-out from German Higher Education institutions. European Journal of Education, 49(4), 497-513.

Howell, G.F., \& Buck, J.M. (2012). The Adult Student and Course Satisfaction: What Matters Most?. Innovative Higher Education, 37(3), 215-226.

$\mathrm{Hu}$, S. (2011). Reconsidering the Relationship Between Student Engagement and Persistence in College. Innovative High Education, 36(2), 97-106. doi: 10.1007/s10755-010-9158-4.

Kucel, A., \& Vilalta-Buf, M. (2013). Why do tertiary education graduates regret their study program? A comparison between Spain and the Netherlands. High Education, 65(5), 565579. https://doi.org/10.1007/s10734-012-9563-y.

Lassibille, G., \& Navarro-Gómez, L. (2008). Why do higher education students drop out? Evidence from Spain. Education Economics, 16(1), 89-105. http://dx.doi.org/10.1080/09645290701523267.

Loureiro, E., Ferreira, M.A., Fresta, M., Ismail, M., Rehman, S.U., \& Broome, M. (2017). Teaching and assessment of clinical communication skills: Lessons learned from a SWOT analysis of Portuguese Angolan and Mozambican Medical Education. Porto Biomedical Journal, 2(2), 47-58. https://doi.org/10.1016/j.pbj.2016.12.005.

Markova, T., Glazkova, I., \& Zaborova, E. (2017). Quality Issues of Online Distance Learning. Procedia - Social and Behavioral Sciences, 182, 685-691.

Indicadores y control estadístico para el seguimiento y evaluación de preferencias de aprendizaje de estudiantes universitarios. Olga María Alegre de la Rosa y Luis Miguel Villar Angulo. 
Marra, R.M., Tsai, Ch.-L., Bogue, B., \& Pytel, J.L. (2015). Alternative Pathways To Engineering Success -Using Academic And Social Integration To Understand Two-Year Engineering Student Success. American Journal of Engineering Education, 6(2), 69-83. Doi:10.1145/2788451.

Mihaljevic, M. (2008). Examining the Efficiency of Croatian Higher Education: An Application of Student Attainment Modeling. Eastern European Economics, 46(1), 74-88. Doi: 10.2753/EEE0012-8775460105.

Morris, L.V. (2012). All Together Now: Degree Completions!. Innovative Higher Education, 37(3), 167-169. Doi:10.1007/s10755-012-9220-5.

Nadelson, L.S., Semmelroth, C., Martinez, G., Featherstone, M., Fuhriman, C.A., \& Sell, A. (2013). Why Did They Come Here? - The Influences and Expectations of First-Year Students' College Experience. Higher Education Studies, 3(1), 50-62. http://dx.doi.org/10.5539/hes.v3n1p50.

Nelson, K., Quinn, C., Marrington, A., \& Clarke, J. (2012). Good practice for enhancing the engagement and success of commencing students. Higher Education, 63(1), 83-96. https://Doi.org/10.1007/s10734-011-9426-y.

Pérez, F., Aldás, J. (eds.), Aragón, R., \& Zaera, I. (2016). U-Ranking 2016. Synthetic Indicators of Spanish Universities. Fourth edition, March 2016. Valencia: Instituto Valenciano de Investigaciones Económicas.

Pike, G.R., Hansen, M.J., \& Childress, J.E. (2014). The Influence Of Students' Pre-College Characteristics, High School Experiences, College Expectations, And Initial Enrollment Characteristics On Degree Attainment. J. College Student Retention, 16(1), 1-23. https://Doi.org/10.2190/CS.16.1.a.

Rapport, M.J.K., Stelzner, D., \& Rodriguez, J (2007). The Doctor of Physical Therapy Degree: A New Curriculum for a New Degree. Physical Disabilities: Education and Related Services, 26(1), 63-76.

Raynor, K. (2016). Book Review: Determined to Succeed? Performance versus Choice in Educational Attainment. Teaching Sociology, 43(1), 70-71. Doi: 10.1177/0092055X.

Richardson, J.T.E. (2012). The attainment of White and ethnic minority students in distance education. Assessment \& Evaluation in Higher Education, 37(4), 393-408. http://dx.Doi.org/10.1080/02602938.2010.534767.

Richardson, J.C., Koehler, A.A., Besser, E.D., Caskurlu, S., Lim, J., \& Mueller, Ch.M. (2015). Conceptualizing and Investigating Instructor Presence in Online Learning Environments. International Review of Research in Open and Distributed Learning, 16(3), June. http://dx.doi.org/10.19173/irrodl.v16i3.2123.

Romero-Gutierrez, M., Jimenez-Liso, M.R., \& Martinez-Chico, M. (2016). SWOT analysis to evaluate the programme of a joint online/onsite master's degree in environmental education

Indicadores y control estadístico para el seguimiento y evaluación de preferencias de aprendizaje de estudiantes universitarios. Olga María Alegre de la Rosa y Luis Miguel Villar Angulo. 
through the students' perceptions. Evaluation and Program Planning, 54, 41-49. Doi:10.1016/j.evalprogplan.2015.10.001.

Stevenson, J. (2012). An Exploration of the Link between Minority Ethnic and White Students' Degree Attainment and Views of Their Future 'Possible Selves'. Higher Education Studies, 2(4), 103-113.

Tashakkori, A., \& Teddlie, C. (2010). Mixed Methods in Social \& Behavioral Research (Second Edition). Washington: Sage.

Thomas, S., Chie, Q.T., Abraham, M., Raj, S.J., \& Beh, L.S. (2014). A Qualitative Review of Literature on Peer Review of Teaching in Higher Education: An Application of the SWOT Framework. Review of Educational Research, 84(1), 112-159. Doi:10.1007/s10734-0079093-1.

Troiano, E., \& Elias, M. (2014). University access and after: explaining the social composition of degree programmes and the contrasting expectations of students. Higher Education, 67(5), 637-654. https://doi.org/10.1007/s10734-013-9670-4.

Vayre, E., \& Vonthron, A.M. (2017). Psychological Engagement of Students in Distance and Online Learning: Effects of Self-Efficacy and Psychosocial Processes. Journal of Educational Computing Research, 55(2), 197-218. https://doi.org/10.1177/0735633116656849.

Villar-Aguilés, A., Hernàndez i Dobon, F.J., \& García-Ros, R. (2017). Reubicándose en la universidad. Propuesta de una tasa de reubicaciones a partir de un estudio de trayectorias educativas. RELIEVE, 23(1), art. 5, 1-11. http://Doi.org/10.7203/relieve.23.1.9059.

Villar, L.M., \& Alegre, O.M. (2008). Measuring Faculty Learning in Curriculum and Teaching Competence (CTC) Online Courses. Interactive Learning Environments, 16(2), 169-181. Doi: 10.1080/10494820701365937.

Wright, S. L., Jenkins-Guarnieri, M.A., \& Murdock, J.L. (2012). Career Development Among FirstYear College Students: College Self-Efficacy, Student Persistence, and Academic Success. $\begin{array}{llll}\text { Journal of Career } & \text { Development, }\end{array}$ https://Doi.org/10.1177/0894845312455509. 